\title{
Use of spatiotemporal templates for pathway discrimination in peripheral nerve recordings: A simulation study
}

\author{
Ryan G. L. Koh ${ }^{1,2}$, Adrian I. Nachman ${ }^{1,3,4}$, and José Zariffa ${ }^{1,2,3}$ \\ ${ }^{1}$ Institute of Biomaterials and Biomedical Engineering, University of Toronto, Toronto, ON M5S 3G4, Canada \\ ${ }^{2}$ Toronto Rehabilitation Institute - University Health Network, Toronto, ON, M5G 2A2 \\ ${ }^{3}$ Edward S. Rogers Sr. Department of Electrical and Computer Engineering Toronto, ON M4G 3V9, Canada \\ ${ }^{4}$ Department of Mathematics, University of Toronto, Toronto, ON M5S 2E4, Canada \\ E-mail: ryan.koh@mail.utoronto.ca
}

\begin{abstract}
Objective: Extraction of information from the peripheral nervous system can provide control signals in neuroprosthetic applications. However, the ability to selectively record from different pathways within peripheral nerves is limited. We investigated the integration of spatial and temporal information for pathway discrimination in peripheral nerves using measurements from a multi-contact nerve cuff electrode. Approach: Spatiotemporal templates were established for different neural pathways of interest, and used to obtain tailored matched filters for each of these pathways. Simulated measurements of compound action potentials propagating through the nerve in different test cases were used to evaluate classification accuracy, percentage of missed spikes, and ability to reconstruct the original firing rates of the neural pathways. Main Results: The mean Pearson correlation coefficients between the original firing rates and estimated firing rates over all tests cases was found to be $0.832 \pm 0.161,0.421 \pm 0.145,0.481 \pm 0.340$ for our algorithm, Bayesian spatial filters, and velocity selective recordings respectively. Significance: The proposed method shows that the spatiotemporal templates were able to provide more robust spike detection and reliable pathway discrimination than these existing algorithms.
\end{abstract}

\section{Introduction}

The identification of motor and sensory information from the bioelectric activity of peripheral nerves has the potential to provide valuable control signals for a range of neuroprosthetic devices, ranging from prosthetic limbs to closed-loop functional electrical stimulation devices [1]. However, selectively recording from specific neural pathways in peripheral nerves is currently a major challenge, especially if the device must be appropriate for chronic implantation in humans.

Techniques or devices that could improve our ability to discriminate the bioelectric activity of multiple sources within a peripheral nerve would provide numerous advantages. Such technology would make for easier characterization of control signals being exchanged between the central nervous system and the targeted limb or organ. Additionally if discrimination could be performed in real-time, this information exchange could be monitored, interpreted and used as a control signal in a neural prosthesis. 
Peripheral nerve recordings can be captured using two broad categories of electrodes: intraneural and extraneural electrodes. Intraneural electrodes penetrate the nerve and thus provide better spatial selectivity and signal-to-noise ratio than their extraneural counterparts. However, because they penetrate the nerve they are much more likely to damage the neural tissue. Extraneural electrodes on the other hand do not penetrate the nerve, minimizing the risk of damage [1].

Nerve cuff electrodes are a type of extraneural electrodes that have been shown to be safe for chronic implantation in humans [2]-[4] but have relatively poor recording selectivity. This has led to various variants in designs (i.e., Flat Interface Nerve Electrode (FINE) [5] [6]) and techniques to try to improve their ability to extract information from the peripheral nerve, while still taking advantages of the safe chronic implantation possibilities that they provide. While early nerve cuff designs provided a single channel of information, a number of multi-contact designs have since been investigated [3] [7] [9]. Several groups have tried to differentiate bioelectric activity within the nerve from nerve cuff recordings [10]. Some have considered using the spatial information (i.e. variations in the signals obtained simultaneously at several recording contacts) through a source localization approach [7][11][12], and through a beamforming approach [8] [13] [14]. Others have proposed using the temporal information (i.e. propagation of a signal between contacts over time) to extract velocity information to infer the fiber type [9] [15]-[17]. These different strategies have been investigated in isolation from each other, but an integrated approach has not been explored. More recently studies focusing on spike sorting in multi-electrode cortical recordings have started to incorporate spatial and temporal information to tackle problems conceptually related to our peripheral nerve problem. Dragas et al. have incorporated spatial and temporal overlap in neuron activity in spike sorting hardware to perform spike detection [18]. Franke et al. have also similarly incorporated the spatial and temporal information to resolve overlapping spikes from neurons in the brain [19].

Here we present a method for discriminating bioelectric activity within the peripheral nerve, where we approach the problem by integrating the spatial and temporal information from a multi-contact nerve cuff electrode into spatiotemporal templates for source identification.

\section{Methods}

Throughout the rest of the paper we use the term "neural pathways" to refer not to an individual nerve fiber, but 
rather to a small group of neighboring nerve fibers associated with a particular function (e.g. a motor command to a single muscle, a cutaneous input from a region of interest, a proprioceptive input form a single muscle, etc.). This functional rather than anatomical definition is beneficial for more easily gauging the clinical implications of the method proposed herein.

The approach we employed was to encode the spatiotemporal characteristics of the recordings from different neural pathways into templates. These templates were obtained from a training set and corresponded to the spatiotemporal "fingerprints" of the different neural pathways of interest. They were then used to classify new recordings in 5 different test scenarios into their corresponding neural pathways.

These 5 test scenarios were:

- Afferent pathways in different fascicles with different fiber types $\left(\mathrm{DF}_{\mathrm{a}} \mathrm{DF} \mathrm{F}_{\mathrm{i}}\right)$

- Afferent pathways in different fascicles with the same fiber type $\left(\mathrm{DF}_{\mathrm{a}} \mathrm{SF}_{\mathrm{i}}\right)$

- Afferent pathways within the same fascicle, with different fiber types. $\left(\mathrm{SF}_{\mathrm{a}} \mathrm{DF}_{\mathrm{i}}\right)$

- Afferent pathways within the same fascicle, with the same fiber type. $\left(\mathrm{SF}_{\mathrm{a}} \mathrm{SF}_{\mathrm{i}}\right)$

- Afferent and efferent pathways within the same fascicle with the same fiber type (SFAE).

The following sections will present our process for creating the spatiotemporal template and using them for pathway discrimination, the finite element (FE) modelling process used to generate the simulated measurements, the different algorithms and the procedures used to evaluate our methods.

\subsection{Spatiotemporal Templates}

In order to construct the template for a given pathway, compound action potentials (CAPs) from that pathway were generated, and the simulated recordings obtained from $M$ contacts at $T$ consecutive time samples as the CAP traverses the nerve cuff were assembled into an $M x T$ matrix. This matrix was then reshaped into a column vector, which constitute the spatiotemporal template for that pathway (Figure 1). Templates were defined in this way for each of the $N$ neural pathways in a given test scenario. The spatiotemporal template for each pathway was used to create a tailored matched filter for that pathway. Since each template corresponds to a specific neural pathway, the identification of the correct template results in pathway discrimination. 


\subsection{Model}

An FE model was created consisting of a rat sciatic nerve surrounded by a 56 -channel multi-contact nerve cuff electrode in a saline bath. The FE model was created by tracing the cross-section a rat sciatic nerve from a histological image obtained in a previous study [20] (Figure 2(a)). The section imaged corresponds to a location slightly proximal to the branching of the nerve, such that distinct fascicles are visible. The conductivities of the different materials in the nerve model are given Table I and were based on related models described in the literature. The 56 electrode contacts were placed on the inside of the nerve cuff and organized in 7 rings of 8 electrodes each, with the dimensions and contact placement based on an existing "matrix" cuff electrode (Figure 2(b)) designed by Schuttler et al. [21]. The ratio of the bath and the nerve dimensions were large enough to avoid boundary effects [22]. The finite element computation of the leadfields was conducted using the SCIRun computing environment [23]. The procedure for obtaining the leadfield from the FE model is described by Weinstein et al. [24].

\subsection{Algorithms}

Three algorithms were used in this study to evaluate their CAP classification accuracy and their ability to reconstruct firing rates.

Our proposed method incorporates the spatiotemporal templates as described in Section 2.1. and uses them to create tailored matched filters corresponding to each neural pathway. Each matched filter is then applied to the time series under test, using a sliding window of 100 samples that has been reshaped into a column vector in the same way as the spatiotemporal templates. Spike detection is performed on the matched filter outputs (see Section 2.4) and the resulting peaks are used for spike classification: the pathway whose corresponding filter has the highest maximum for a given spike is chosen as the active pathway. The spike classification was based on the maximum filter output of the tailored matched filters at the detected spikes after dividing by their corresponding normalization factor $\mathbf{u}_{\mathrm{i}}$.

$$
\begin{gathered}
\boldsymbol{u}_{i}=\left\|\boldsymbol{L}_{i}\right\|_{2} \\
i=1: N
\end{gathered}
$$

Where $\mathbf{L}_{\mathrm{i}}$ is the spatiotemporal column vector of the $\mathrm{i}^{\text {th }}$ neural pathway.

One of the comparison algorithms was the velocity selective recording (VSR) method by Metcalfe et al. [15] [17] which utilizes the temporal information. This method uses a "delay-and-add" operation where recordings at constant 
distances can be added together with a delay factor. Recordings that correspond to a CAP with a conduction velocity that matches the chosen delay will result in constructive interference (signal enhancement) whereas noise and other sources of interference will lead to destructive interference.

The other comparison algorithm was the Bayesian Spatial Filter (BSF) method by Tang et al. [8] which relies on spatial information. The Bayesian spatial filter method is a modification of the Champagne algorithm [25] where not only the background noise and interferences are suppressed, but the spatial filter for each neural pathway of interest also suppresses the activity of the other target pathways. This approach results in tailored spatial filter designed for each signal of interest.

In this paper, recordings resulting from CAPs from different neural pathways that overlapped in time were ignored. All overlapping cases were placed in a separate class (approximately $7.5 \%$ of the test set) that was not investigated by any of the 3 algorithms.

\subsection{Training and Evaluation}

5000 myelinated mammalian fiber APs were created using the Neurocal Matlab toolbox (Case Western Universiy, Ohio, USA) based on the Schwarz-Reid-Bostock model. Note that although this model simulates a single-fiber AP, we made the simplifying assumption here that it could be used to approximate the shape of a CAP propagating through the FE model. In order to study the effects of having non-ideal templates as well as model the stochastic nature of different fibers activating for a particular function, we introduced variability in our simulated CAPs by introducing small changes in three parameters: the shape of the CAP, the conduction velocity, and the spatial location. For the shape variations, the $E_{k}$ and $E_{N a}$ values were changed to randomly selected values at step sizes of $0.1 \mathrm{mV}$ between $-84.2 \mathrm{mV}$ to $-83.8 \mathrm{mV}$ and $49.8 \mathrm{mV}$ to $50.2 \mathrm{mV}$ respectively. The CAPs were propagated at various spatial locations within each neural pathway, by randomly selecting a mesh element from within the chosen region of the fascicle (as shown in Figure 3a), for each trial. The conduction velocities were also slightly varied depending on the fiber types in a given test scenario (Figure 3). The velocities ranged from $93.74 \mathrm{~m} / \mathrm{s} \mathrm{to} 95.98 \mathrm{~m} / \mathrm{s}$ (A $\alpha$ fiber), $63.61 \mathrm{~m} / \mathrm{s}$ to $65.84 \mathrm{~m} / \mathrm{s}$ (A $\beta$ fiber) and $23.99 \mathrm{~m} / \mathrm{s}$ to $26.23 \mathrm{~m} / \mathrm{s}$ (A $\delta$ fiber) corresponding to fiber diameters at step size of $0.1 \mu \mathrm{m}$ from $16.8 \mu \mathrm{m}$ to $17.2 \mu \mathrm{m}, 11.4 \mu \mathrm{m}$ to $11.8 \mu \mathrm{m}, 4.3 \mu \mathrm{m}$ to $4.7 \mu \mathrm{m}$ respectively.

For the different fiber type cases (i.e. $\mathrm{DF}_{a} \mathrm{DF}_{\mathrm{i}}$ and $\mathrm{SF}_{\mathrm{a}} \mathrm{DF} \mathrm{F}_{\mathrm{i}}$ ), the simulations included 3 neural pathways, and each 
fiber type (i.e. $\mathrm{A} \alpha, \mathrm{A} \beta, \mathrm{A} \delta$ ) was used in one of the neural pathways. For the same fiber type cases (i.e. $\mathrm{DF}_{\mathrm{a}} \mathrm{SF} \mathrm{F}_{\mathrm{i}}$, $\mathrm{SF}_{\mathrm{a}} \mathrm{SF}_{\mathrm{i}}$ ), the simulations again included 3 neural pathways, but all neural pathways were of a single fiber type. For the SFAE case, the simulations included 2 neural pathways (one afferent and one efferent). The A $\delta$ fiber properties were used for both the afferent and efferent CAPs, because the values selected also approximate the characteristics of an efferent $\mathrm{A} \gamma$ fiber, and using the same fiber characteristics ensures that any discrimination will be based on direction rather than conduction velocity.

The resulting CAPs were used first to quantify the classification accuracy of each of the three algorithms on a spike-by-spike basis. The generated CAPs were used in a 5-fold cross-validation process (in which CAPs were divided into 5 consecutive sets of 1000 CAPs, i.e. CAPs from 1-1000, 1001-2000, .., 4001-5000). The training sets were used to obtain the spatiotemporal templates for each neural pathway in a given test scenario. The templates were 3.33 ms long (i.e. 56 channels x 100 time samples sampled at $30 \mathrm{kHz}$ ) and built from all CAPs in the training set. Time series that combined the activity from the 3 neural pathways with constant firing rates ranging between 35 to $50 \mathrm{~Hz}$ (or 2 pathways in the SFAE case) were created using the CAPs in the testing set, for the different test cases. White Gaussian noise was added to each of the time series, as well as to the training set, corresponding to decreasing levels of signal-to-noise ratios (SNR) (i.e. $[\infty,-5,-10,-15,-20,-25] \mathrm{dB}$ ) defined based on the signal power in the entire time series, implemented using the awgn function in Matlab (Figure 3(b)). The recordings were sampled at 30 $\mathrm{kHz}$ and tripole referenced [26].

Spike detection was done using the findpeaks function in Matlab by finding peaks within all matched filtered outputs with a minimum height of 4 standard deviations $(\sigma)$ of the noise and with a peak prominence of $5 \sigma$ of the noise. Resulting spikes were then filtered by removing any spikes below a threshold, which was set to $1.5 \sigma$ of all detected spike values. In the case of $\mathrm{SNR}=\infty$, the thresholds were set manually based on the matched filter outputs for a small portion of data for each test scenario. Spikes that were not detected by any of the tailored matched filters were considered as missed.

Similar to our algorithm, spike detections were performed on the output of the VSR and BSF methods, where detected spikes were based on a minimum height of $3 \sigma$ of the noise. Spike classification for the VSR method was based on taking the maximum detected value of the different pathways. For the BSF method, classification was based on the highest Euclidean norm computed from a $3.33 \mathrm{~ms}$ long vector (i.e. 100 time sample vector corresponding to 
49 points before and 50 points after each detected spike location) using the spatial filter outputs tailored for a given pathway.

The second metric investigated was the ability of the algorithms to reconstruct the firing rate of each pathway. Reconstruction of the estimated firing rate was performed using all CAPs generated for the spatiotemporal templates. A new time series was created, where the CAP timings were determined using a Poisson process whose average firing rate varied between 0 and $60 \mathrm{~Hz}$ according to a modulating envelope for each test scenario. The firing rates were calculated using the convolution of the estimated spike trains with a Gaussian kernel with a standard deviation of $150 \mathrm{~ms}$ [28]. The Pearson correlation coefficient was used to measure how correlated the original firing rates were to the estimated firing rates.

We further sought to determine the tradeoff between performance and the number of electrode contacts. The performance of the algorithms was investigated across different configurations corresponding to 3 rings of 8 contacts (3x8), 5 rings of 8 contacts $(5 \times 8)$ and 7 rings of 8 contacts $(7 \times 8)$. The same parameters were used as described in the sections above, however for concision this analysis was only conducted at an SNR of -10 dB, which corresponds well to expected in-vivo signal quality.

Lastly, given that our templates were based on all of the CAPs in the training set, we investigated how performance might vary if the CAPs used to construct the template had to first be identified through spike detection, as would be the case in vivo. Using the $\mathrm{DF}_{\mathrm{a}} \mathrm{DF}_{\mathrm{i}}$ case, the normalized root mean square error (NRMSE) was calculated between the spatiotemporal template constructed using all CAPs in training set, compared to one constructed using the spikes extracted from the time series through spike detection (which may miss some CAPs as well as include spurious ones). The 8 contacts in the center ring were averaged for each SNR case $(-5,-10,-15,-20,-25 \mathrm{~dB})$ and manual thresholding was used to extract spikes. The templates were normalized by their $1^{2}$ norms in order to focus on shape differences, and the RMSE was calculated. The classification accuracies using these templates were also calculated.

\section{Results}

\subsection{Classification}

Figure 4 shows the mean classification accuracies from the 5 folds of the cross-validation process, for each of the 
first four test cases for all algorithms over the various SNRs. Figure 4(a) reveals that in the $\mathrm{DF}_{\mathrm{a}} \mathrm{DF} \mathrm{F}_{\mathrm{i}}$ case our algorithm was able to differentiate between the different neural pathways quite consistently at all of the SNRs with very high classification accuracy compared to the VSR and the BSF approaches. A similar result can be seen in the $\mathrm{SF}_{\mathrm{a}} \mathrm{DF}_{\mathrm{i}}$ case (Figure 4(b)). The mean classification accuracies over the $6 \mathrm{SNRs}$ for the $\mathrm{DF}_{\mathrm{a}} \mathrm{DF}_{\mathrm{i}}$ case were $84.9 \pm 14.7 \%, 20.8 \pm 19.2 \%$, and $41.6 \pm 23.2 \%$ for our algorithm, the BSF method and the VSR method respectively. The ranges of values observed for the three methods were from 54.2-92.5\%, 1.9-60.0\%, and 0.0-61.9\%, respectively. In the $\mathrm{SF}_{\mathrm{a}} \mathrm{DF}$ i case, the mean classification accuracies were $85.5 \pm 13.1 \%, 18.2 \pm 14.8 \%$, and $41.7 \pm 21.8 \%$ for our algorithm, the BSF method and the VSR method respectively. The ranges of values observed for the three methods were from $54.0-92.5 \%, 1.4-52.6 \%$, and $0.0-61.8 \%$, respectively.

Figure 4(c) shows that in the $\mathrm{DF}_{\mathrm{a}} \mathrm{SF}_{\mathrm{i}}$ case our algorithm was less successful at classifying the different neural pathways but was still able to provide a better prediction of the spikes classes than the other 2 algorithms at almost all SNRs. It can also be clearly seen that our algorithm was able to perform better as the speed of the fiber decreased (i.e. $\mathrm{A} \alpha \rightarrow \mathrm{A} \beta \rightarrow \mathrm{A} \delta$ ). The mean classification accuracies in the $\mathrm{DF}_{\mathrm{a}} \mathrm{SF}_{\mathrm{i}}$ case over all fiber types were $45.3 \pm 17.9 \%$, $12.9 \pm 11.8 \%$, and $21.1 \pm 12.6 \%$ for our algorithm, the BSF method and the VSR method respectively. The ranges of values observed for the three methods were from $26.8-80.3 \%, 1.4-76.0 \%$, and $0.0-37.2 \%$ respectively.

Figure 4(d) shows that in the $\mathrm{SF}_{\mathrm{a}} \mathrm{SF}_{\mathrm{i}}$ case all algorithms were unable to discriminate between the different neural pathways sufficiently on average. The mean classification accuracies in this case over all fiber types were $31.4 \pm 1.4 \%, 9.5 \pm 11.8 \%$, and $19.7 \pm 14.0 \%$ for our algorithm, the BSF method and the VSR method respectively. The ranges of values observed for the three methods were from $25.2-39.4 \%, 1.4-63.6 \%$, and $0.0-33.5 \%$, respectively.

Figure 5 shows the mean fraction of missed spikes in the first four test cases. The figure reveals that the BSF and VSR method had difficulty detecting spikes at the lower SNR levels (i.e. $-20 \mathrm{~dB}$ ) and that our algorithm was able to more accurately detect spikes across the different SNR levels. The mean percentages of missed spikes over the first four test cases were $2.4 \pm 6.3 \%, 56.0 \pm 35.3 \%, 31.5 \pm 40.4 \%$ for our algorithm, the BSF method and the VSR method respectively. The ranges of values observed for the three methods were from $0.0-33.2 \%, 0.0-94.6 \%$, and $0.0-99.9 \%$, respectively. This clearly indicates that the spatiotemporal templates can be used as a more robust method for spike detection than a traditonal thresholding method.

Lastly Figure 6 shows the results of the SFAE case. It can be seen that the activity can be differentiated well by 
both our algorithm and the VSR method but our algorithm was able to perform much better as the SNR dropped. The mean classification accuracies in this case were $95.8 \pm 0.3 \%, 18.9 \pm 19.7 \%, 56.3 \pm 37.7 \%$ for our algorithm, the BSF method and the VSR method respectively. The ranges of values observed for the three methods were from 94.9.95.9\%, 4.0-57.5\%, and 0.1-95.0\%, respectively. The mean percentages of missed spikes for the SFAE case were $0.0 \pm 0.1 \%, 62.3 \pm 39.2 \%, 40.6 \pm 44.3 \%$ for our algorithm, the BSF method and the VSR method respectively. The range of values observed for the three methods was from $0.0-0.4 \%, 0.0-93.0 \%$, and $0.0-99.5 \%$, respectively.

\subsection{Reconstruction}

Figure 7 shows the mean Pearson correlation coefficients between the original firing rates and the estimated firing rates for the different test cases. The mean coefficients over all test cases were $0.832 \pm 0.161,0.421 \pm 0.145$, $0.481 \pm 0.340$ for our algorithm, the BSF method and the VSR method respectively. The range of values observed for the three methods was from $0-1.00,-0.103-0.999$, and $0-1.00$, respectively. Note that the correlation coefficient was set to 0 if no spikes were assigned to that class.

Figure 8 shows an example of the original firing rate of the 3 different neural pathways and the reconstructed firing rates predicted by the 3 algorithms in the $\mathrm{DF}_{\mathrm{a}} \mathrm{DF}_{\mathrm{i}}$ case. It can be seen in the figure that our algorithm was able to capture the firing rates in the 3 different neural pathways more accurately compared to the BSF and VSR methods. As the SNR drops our algorithm was still able to capture the firing rate consistently and fairly accurately. These figures also reveal that the general shape of the firing rate can be estimated sufficiently even with some misclassifications of spikes. Figure 9 shows an example of the predicted firing pattern of each pathway using our algorithm for a sub-section of the $\mathrm{DF}_{\mathrm{a}} \mathrm{DF}_{\mathrm{i}}$ case.

\subsection{Influence of the number of contacts on performance}

Table II shows the results of the $\mathrm{DF}_{a} \mathrm{DF}_{\mathrm{i}}, \mathrm{DF}_{\mathrm{a}} \mathrm{SF}_{\mathrm{i}}, \mathrm{SF}_{\mathrm{a}} \mathrm{DF}_{\mathrm{i}}, \mathrm{SF}_{\mathrm{a}} \mathrm{SF}_{\mathrm{i}}$ cases for all 3 arrangements, the $3 \times 8$ configuration, $5 \times 8$ configuration, and $7 \times 8$ configuration for $\mathrm{SNR}=-10 \mathrm{~dB}$. This table shows a general trend towards decreasing performance as the number of contacts is reduced, but also suggests that the performance of the spatiotemporal templates is more robust to decreasing numbers of contacts than the other two algorithms examined. 


\subsection{Influence of non-ideal spike detection on performance}

Table III shows the NRMSE calculated for the neural pathway templates in the $\mathrm{DF}_{\mathrm{a}} \mathrm{DF}_{\mathrm{i}}$ case for all folds over all SNRs except SNR $=\infty$, along with their associated classification accuracies. For comparison, the classification accuracies when using all CAPs in the training set are provided in Figure 4a. Table III also shows how many of the 4000 true CAPs were detected in each case, and what the proportion of true and spurious CAPs was after spike detection. This table reveals that the templates constructed based on a spike detection process are very similar to those calculated based on known spike times, and that their classification performance does not start to drop until the SNR goes below $-20 \mathrm{~dB}$.

\section{Discussion}

The classification results seen from the different test cases suggest that both the spatial and temporal information play an important role in the ability to discriminate between different neural pathways. The temporal information would seem to play a larger role in this discrimination process. This can be seen more clearly from the classification results in the $\mathrm{DF}_{\mathrm{a}} \mathrm{DF}_{\mathrm{i}}$ and $\mathrm{SF}_{\mathrm{a}} \mathrm{DF}_{\mathrm{i}}$ cases which show relatively similar results in classification, and contrast strongly with the classification results from the $\mathrm{DF}_{\mathrm{a}} \mathrm{SF}_{\mathrm{i}}$ and $\mathrm{SF}_{\mathrm{a}} \mathrm{SF}_{\mathrm{i}}$ cases. The ability to discriminate between neural pathways decreased due to the loss of the temporal information provided by the different conduction velocities of different fiber types. We can also see a drop in classification accuracy between the $\mathrm{DF}_{\mathrm{a}} \mathrm{SF}_{\mathrm{i}}$ and $\mathrm{SF}_{\mathrm{a}} \mathrm{SF}_{\mathrm{i}}$ cases. It is clear that the spatial information also plays a role in the discrimination of these neural pathways, but less so than the temporal information.

Furthermore, as observed in Figure 4-Figure 6, we can see that our algorithm was able to not only detect the spikes more reliably but also classify and reconstruct the firing rates better than the comparison algorithms in most of the test cases. This reinforces our hypothesis that incorporating both the spatial and temporal information is vital in the discrimination of different neural pathways in the peripheral nerve.

In the $\mathrm{SF}_{\mathrm{a}} \mathrm{SF}_{\mathrm{i}}$ case, the results show low classification accuracies from all algorithms tested and this suggests that our techniques currently are not adequate for distinguishing different neural pathways that are active within the same fascicle and that are of the same fiber type. Further investigation is needed to conclusively determine whether discrimination in this scenario is possible using a nerve cuff. 
Additionally, in the $\mathrm{DF}_{\mathrm{a}} \mathrm{DF}_{\mathrm{i}}$ or $\mathrm{SF}_{\mathrm{a}} \mathrm{DF}_{\mathrm{i}}$ case we can see that the decrease in classification for lower SNR was likely due to missed spikes rather than misclassification, given that the increase in the percentage of missed spikes corresponded closely to the decrease in classification accuracy. This suggests that correct spike classes are identified in detected spikes but better spike detection methods are needed as the SNR drops to decrease the amount of missed spikes.

It is important to note that we have used a sampling frequency of $30 \mathrm{kHz}$ in our test trials, and this time resolution may have impaired the VSR method's ability to discriminate between all 3 pathways (in [17], a sampling rate of $500 \mathrm{kHz}$ was used to detect velocities in the range of $5-20 \mathrm{~m} / \mathrm{s}$ ). Given a higher sampling frequency the VSR method may match the classification accuracies of our algorithm in the $\mathrm{DF}_{\mathrm{a}} \mathrm{DF}_{\mathrm{i}}$ and $\mathrm{SF}_{\mathrm{a}} \mathrm{DF}_{\mathrm{i}}$ case. Additionally, given a higher sampling frequency our algorithm may also be able to discriminate the neural pathways more effectively in the $\mathrm{DF}_{\mathrm{a}} \mathrm{SF}_{\mathrm{i}}$ cases involving $\mathrm{A} \alpha$, and $\mathrm{A} \beta$ fibers types and could likely reach similar classification accuracies as seen in the A $\delta$ case. The $30 \mathrm{kHz}$ sampling frequency was chosen here to remain consistent with the capabilities of most commercial multi-channel neural data acquisition systems.

The classification results from the BSF method may likely have been higher and more similar to results reported by Tang et al. if the test signals were to undergo preprocessing techniques (such as rectify-bin-integration) to enhance the SNR before applying the BSFs. No preprocessing techniques were used in this case to keep the test conditions the same for all algorithms as no preprocessing was applied for our algorithm and the VSR method. In addition, a different metric other than the Euclidean norm may be more appropriate and lead to better results for the BSF method. The Euclidean norm was used here to decrease the effects of noise by obtaining an average value. Furthermore, the performance may also have been affected by the geometry of the electrode, as in this study a cylindrical cuff electrode was used versus the rectangular geometry of the FINE used in [8].

Our results additionally suggest that good performance may be achievable with fewer rings. For example, Table II shows that the use of the $5 \times 8$ configuration resulted in only minor performance drops in most cases for our algorithm, and a small increase in the $\mathrm{DF}_{\mathrm{a}} \mathrm{DF}_{\mathrm{i}}$ case. Therefore, a more compact multi-contact nerve cuff design could potentially be implemented (e.g. 5 rings of 8 contacts, or even 5 rings of 4 contacts, since the spatial information seems to play a smaller role in the discrimination of the neural pathways). This may allow for shorter nerve cuffs that would allow access to a larger variety of peripheral nerves when used for in vivo studies. However, further work is required to 
understand how the layout of the contacts and the geometry of the electrode can be selected in order to achieve the best trade-off between the complexity of the device and the discriminative performance of the spatiotemporal patterns.

In practice, the need to collect the proposed spatiotemporal templates for the pathways of interest will be a disadvantage, but given that the improvement in discrimination is significant this tradeoff does not seem unreasonable. For in vivo situations, the spatiotemporal templates could be obtained experimentally through a calibration step. For example, in the rat model of the sciatic nerve one could selectively activate the tibial, peroneal and sural branches by performing dorsiflexion, plantarflexion and pricking of the heel, respectively, then use traditional spike detection to locate spikes that can be averaged to create the template for each neural pathway. In humans, the user could be asked to voluntary attempt to contract certain muscles, and/or provided with sensory stimuli (e.g. passive joint movement, cutaneous mechanical stimulation, etc.) to elicit activation in certain pathways in order to define spatiotemporal templates for all the pathways of interest. These spatiotemporal templates could thereafter be used for spike detection and classification to track the firing rates of individual pathways, which could then be used as command signals in neuroprostheses. Lastly, if one were to use these spatiotemporal templates in practice it would be advisable to base the training set on the natural activity of the pathways of interest, rather than direct stimulation. This will ensure the training set is more closely related to the activity that one would observe in practice. While the spatiotemporal templates used in this study assumed correct spike detection, the results in Table 3 suggest that templates based on a threshold-based spike detection process would yield very similar performance up to an SNR of approximately $-15 \mathrm{~dB}$ (with template NRMSE on the order of $10^{-4}$ ), thus supporting the feasibility of the calibration process described above. Chronic implantation for selective peripheral nerve recordings has been limited to date, however this process may be accelerated if the discrimination performance can be improved with novel methods such as the one presented here.

\subsection{Limitations}

While the present results provide a proof-of-concept for the integration of the spatial and temporal information, further work is warranted to address cases when CAPs overlap causing recordings that consists of activity from multiple neural pathways, as well as to validate the method in an in-vivo study. 
Other aspects that should also be considered are the effects of other types of noise, such as an electromyogram signal that may occur during the collection of the peripheral nerve recording, and produce more structured interference than the independent white Gaussian noise signals used here. The method must also be investigated in nerve models with larger numbers of fascicles, as human nerves can have many more fascicles than the sciatic nerve model used here, and pathways that are more centrally located.

\section{Conclusion}

This study has shown that the use of spatiotemporal information improves the ability to discriminate between different peripheral neural pathways in various test cases. The spatiotemporal templates allow for robust spike detection at low SNRs. Additionally they provide for more reliable discrimination in the $\mathrm{DF}_{\mathrm{a}} \mathrm{DF}_{\mathrm{i}}, \mathrm{SF}_{\mathrm{a}} \mathrm{DF}_{\mathrm{i}}$ and $\mathrm{SFAE}$ cases at almost all SNRs compared to the VSR and BSF methods. In the $\mathrm{DF}_{\mathrm{a}} \mathrm{SF}_{\mathrm{i}}$ case, they are able to partially discriminate the neural pathways (depending on fiber type) but still provide better discrimination than the VSR and BSF methods at most SNRs. Discrimination in the $\mathrm{SF}_{\mathrm{a}} \mathrm{SF}_{\mathrm{i}}$ case may be possible but will require further development beyond the current techniques. These results demonstrate that leveraging both the spatial and temporal variations in multi-contact nerve cuff recordings leads to improvements over state-of-the-art methods in peripheral nerve recordings.

\section{Acknowledgements}

This work was supported in part by the Natural Sciences and Engineering Research Council of Canada (RGPIN2014-05498 and RGPIN-2016-06329) and the Institute of Biomaterials and Biomedical Engineering at the University of Toronto.

\section{References}

[1] del Valle J and Navarro X 2013 Interfaces with peripheral nerve for the control of neuroprostheses Int. Rev of Neurobio.109 63-83.

[2] Waters R, McNeal W, Faloon W and Clifford B 1985 Functional electrical stimulation of the peroneal nerve for hemiplegia. Long-term clinical follow-up Bone Joint Surg. Am. 67 792-3

[3] Tan D W, Schiefer M A, Keith M W, Anderson J R and Tyler D J 2015 Stability and selectivity of a chronic multi-contact cuff electrode for sensory stimulation in human amputees J. Neural Eng. 12 
[4] Tan D W, Schiefer M A, Keith M W, Anderson J R, Tyler J and Tyler D J 2014 A neural interface provides longterm stable natural touch perception Science Translational Med. 6

[5] Tyler D and Durand D M 2002 Functionally selective peripheral nerve stimulation with a flat interface nerve electrode IEEE Neural Systems and Rehabil. Eng. 10 294-303

[6] Tyler D and Durand D M 2003 Chronic response of the rat sciatic nerve to the flat interface nerve electrode Ann. of Biomed. Eng. 31

[7] Zariffa J , Nagai M K, Schuettler M, Stieglitz T, Daskalakis Z J and Popovic M R 2011 Use of an experimentally derived leadfield in the peripheral nerve pathway discrimination problem IEEE Trans. Neural Syst. Rehabil. Eng $19147-56$

[8] Tang Y, Wodlinger B and Durand D M 2014 Bayesian spatial filters for source signal extraction: A study in the peripheral nerve IEEE Neural Syst. Rehabil. Eng 22 302-11

[9] Schuettler M, Donaldson N, Seetohul V and Taylor J 2013 Fibre-selective recording from the peripheral nerves of frogs using a multi-electrode cuff J. of Neural Eng. $\mathbf{1 0}$

[10] Zariffa J 2014 A review of source separation and source localization approaches in peripheral nerves Signals, Syst. and Comp. Pacific Grove IEEE pp 293-8

[11] Zariffa J and Popovic M R 2008 Application of EEG source localization algorithms to the monitoring of active pathways in peripheral nerves Conf. Proc. IEEE Engineering in Medicine and Biology Society Vancover IEEE pp 4216-9

[12] Zariffa J and Popovic M R 2009 Localization of active pathways in peripheral nerves: A stimulation study IEEE Trans. Neural Syst. and Rehabil. Eng 17 53-62

[13] Wodlinger B and Durand D M 2009 Localization and recovery of peripheral neural sources with beamforming algorithms IEEE Trans Neural Syst. and Rehabil. Eng. 17 461-8

[14] Wodlinger B and Durand D M 2011 Selective recovery of fascicular activity in peripheral nerves J. Neural Eng. 8

[15] Metcalfe B W, Chew D J, Clarke C T, Donaldson N and Taylor J 2014 Fibre-selective discrimination of physiological ENG using velocity selective recordings $36^{\text {th }}$ Annu. Int. Conf. IEEE Engineering in Medicine and Biology Society Chicago pp 2645-8 
[16] Al-Shueli A I, Clarke C T, Donaldson N and Taylor J 2014 Improved signal processing methods for velocity selective neural recordings using multi-electrode cuffs IEEE Trans. Biomed. Circuits and Syst $\mathbf{8}$

[17] Metcalfe B W, Chew D J, Clarke C T, Donaldson N, and Taylor J 2015 A new method for spike extraction using velocity selective recording demonstrated with physiological ENG in rat J. Neurosci. Methods 251 47-55

[18] Dragas J, Jackel D, Hierlemann A and Franke F 2015 Complexity optimization and high-throughput lowlatency hardware implementation of a multi-electrode spike sorting algorithm IEEE Trans. Neural Syst. and Rehabil. Eng. 23 149-58

[19] Franke F, Propper R, Alle H, Meier P, Geiger J R, Obermayer K and Munk M H 2015 Spike sorting of synchronous spikes from local neuron ensembles J. Neurophysiology $1142535-49$

[20] Zariffa J 2009 Bioelectric source localization in peripheral nerves [Ph.D]. Institute of Biomaterials and Biomedical Engineering, University of Toronto

[21] Schuettler M, Triantis I F, Rubehn B and Stieglitz T 2007 Matrix cuff electrodes for fiber and fascicle selective peripheral nerve recording and stimulation $12^{\text {th }}$ Ann. Conf. Int. Functional Electrical Stimulation Society Philadelphia

[22] Yoo P B and Durand D M 2005 Selective recording of the canine hypoglossal nerve using a multicontact flat interface nerve electrode IEEE Trans. Biomed. Eng. 52 1461-9

[23] SCIRun: A Scientific Computing Problem Solving Environment. Scientific Computing and Imaging Institute (SCI), http://www.sci.utah.edu/software/scirun.html. 2015

[24] Weinstien D, Zhukov L and Johnson C 2000 Lead-field bases for electroencephalography source imaging Ann. Biomed. Eng. 28 1059-65

[25] Wipf D P, Owen J P, Attias H T, Sekihara K and Nagarajan S 2010 Robust Bayesian estimation of the location, orientation, and time course of multiple correlated neural sources using MEG NeuroImage 49 641-55

[26] Struijk J J and Morten T 1995 Tripolar nerve cuff recording: stimulus artifact, EMG and the recorded nerve signal $17^{\text {th }}$ Annu. Conf. IEEE Engineering in Medicine and Biology Society Montreal IEEE pp 1105-6

[27] Parrini S, Delbeke J, Romero E, Legat V and Veraart C 1999 Hybrid finite elements and spectral method for computation of the electric potential generated by a nerve cuff electrode Med. Biology Eng. Comput. 37 733-6 
[28] Cunningham J P, Gilja, V, Ryu S I and Shenoy K V 2009 Methods for estimating neural firing rates, and their application to brain-machine interfaces Neural Networks 22 1235-46 
Table 1. Parameters for the FE model of the rat sciatic nerve

\begin{tabular}{|c|c|c|}
\hline Parameter & Values & References \\
\hline Nerve length & $5 \mathrm{~cm}$ & \\
\hline Cuff length & $2.3 \mathrm{~cm}$ & [12] [21] \\
\hline Cuff width & $30 \mu \mathrm{m}$ & [12] [21] \\
\hline Cuff radius & $500 \mu \mathrm{m}$ & [12] [21] \\
\hline Endoneurium conductivity (radial) & $8.26 \times 10^{-2} \mathrm{~S} / \mathrm{m}$ & [12] [22] \\
\hline $\begin{array}{l}\text { Endoneurium conductivity } \\
\text { (longitudinal) }\end{array}$ & $0.571 \mathrm{~S} / \mathrm{m}$ & [12] [22] \\
\hline $\begin{array}{l}\text { Perineurium conductivity } \\
\text { (all directions) }\end{array}$ & $2.1 \times 10^{-3} \mathrm{~S} / \mathrm{m}$ & {$[12][22]$} \\
\hline $\begin{array}{l}\text { Epineurium conductivity } \\
\text { (all directions) }\end{array}$ & $8.26 \times 10^{-2} \mathrm{~S} / \mathrm{m}$ & {$[12][22]$} \\
\hline $\begin{array}{l}\text { Encapsulation tissue conductivity } \\
\text { (all directions) }\end{array}$ & $6.59 \times 10^{-2} \mathrm{~S} / \mathrm{m}$ & [12] [27] \\
\hline Saline conductivity (all directions) & $2 \mathrm{~S} / \mathrm{m}$ & [12] [22] \\
\hline Cuff conductivity (all directions) & $1 \times 10^{-7} \mathrm{~S} / \mathrm{m}$ & [12] [22] \\
\hline
\end{tabular}


Table 2. Classification accuracies at $\mathrm{SNR}=-10 \mathrm{~dB}$

\begin{tabular}{|c|c|c|c|}
\hline $\mathrm{DF}_{\mathrm{a}} \mathrm{DF}_{\mathrm{i}}$ Case & & & \\
\hline Algorithm & $3 \times 8$ Configuration & $5 \times 8$ Configuration & $7 \times 8$ Configuration \\
\hline ST & $72.05 \pm 1.10$ & $90.39 \pm 0.38$ & $92.26 \pm 0.14$ \\
\hline $\mathrm{BSF}$ & $12.30 \pm 1.21$ & $14.37 \pm 2.67$ & $24.77 \pm 2.82$ \\
\hline VSR & $27.1 \pm 0.79$ & $46.95 \pm 0.83$ & $55.51 \pm 0.59$ \\
\hline \multicolumn{4}{|l|}{$\mathrm{DF}_{\mathrm{a}} \mathrm{SF}_{\mathrm{i}}$ Case } \\
\hline Algorithm & $3 \times 8$ Configuration & $5 \times 8$ Configuration & $7 \times 8$ Configuration \\
\hline ST & $47.68 \pm 2.83$ & $50.67 \pm 0.32$ & $47.89 \pm 2.08$ \\
\hline $\mathrm{BSF}$ & $7.40 \pm 2.25$ & $7.05 \pm 2.28$ & $7.32 \pm 3.14$ \\
\hline VSR & $21.17 \pm 7.89$ & $31.73 \pm 1.73$ & $32.55 \pm 0.56$ \\
\hline \multicolumn{4}{|l|}{$\mathrm{SF}_{\mathrm{a}} \mathrm{DF}_{\mathrm{i}}$ Case } \\
\hline Algorithm & $3 \times 8$ Configuration & $5 \times 8$ Configuration & $7 \times 8$ Configuration \\
\hline ST & $73.63 \pm 1.14$ & $90.46 \pm 0.50$ & $92.37 \pm 0.07$ \\
\hline $\mathrm{BSF}$ & $12.30 \pm 1.07$ & $13.28 \pm 1.81$ & $22.37 \pm 4.52$ \\
\hline VSR & $26.15 \pm 0.94$ & $47.58 \pm 0.92$ & $55.92 \pm 0.88$ \\
\hline \multicolumn{4}{|l|}{$\mathrm{SF}_{\mathrm{a}} \mathrm{SF}_{\mathrm{i}}$ Case } \\
\hline Algorithm & $3 \times 8$ Configuration & $5 \times 8$ Configuration & $7 \times 8$ Configuration \\
\hline ST & $31.11 \pm 1.53$ & $30.68 \pm 2.11$ & $31.22 \pm 1.48$ \\
\hline $\mathrm{BSF}$ & $6.82 \pm 2.51$ & $7.09 \pm 2.43$ & $7.24 \pm 2.84$ \\
\hline VSR & $12.29 \pm 3.69$ & $31.58 \pm 1.35$ & $32.66 \pm 0.37$ \\
\hline \multicolumn{4}{|l|}{ SFAE Case } \\
\hline Algorithm & $3 \times 8$ Configuration & $5 \times 8$ Configuration & $7 \times 8$ Configuration \\
\hline ST & $95.69 \pm 0.20$ & $95.62 \pm 0.00$ & $95.83 \pm 0.15$ \\
\hline BSF & $13.78 \pm 3.67$ & $16.29 \pm 6.78$ & $17.77 \pm 8.22$ \\
\hline VSR & $87.07 \pm 5.32$ & $90.48 \pm 3.88$ & $90.34 \pm 3.19$ \\
\hline
\end{tabular}


Table 3. Impact of spike detection on template at different noise levels

\begin{tabular}{cccccc}
\hline Noise level $(\mathrm{dB})$ & NRMSE $\left(\mathrm{x} 10^{-3}\right)$ & $\begin{array}{c}\text { Max Abs. Error } \\
(\%)\end{array}$ & $\begin{array}{c}\text { Number of total } \\
\text { spikes detected }\end{array}$ & $\begin{array}{c}\text { Percent of actual } \\
\text { spikes from detected } \\
(\%)\end{array}$ & $\begin{array}{c}\text { Classification } \\
\text { Accuracy } \\
(\%)\end{array}$ \\
\hline-5 & $0.000 \pm 0.000$ & 0.00 & $4000 \pm 0.00$ & $100 \pm 0.00$ & $92.47 \pm 0.00$ \\
-10 & $0.009 \pm 0.003$ & 2.15 & $3975 \pm 49.47$ & $99.63 \pm 0.02$ & $92.47 \pm 0.00$ \\
-15 & $0.096 \pm 0.025$ & 6.05 & $3255 \pm 567.24$ & $94.66 \pm 0.09$ & $92.09 \pm 0.10$ \\
-20 & $0.545 \pm 0.147$ & 16.82 & $1771 \pm 808.43$ & $73.74 \pm 0.60$ & $80.90 \pm 0.76$ \\
-25 & $1.110 \pm 0.314$ & 24.84 & $820 \pm 131.60$ & $50.14 \pm 0.19$ & $37.55 \pm 0.50$ \\
\hline
\end{tabular}




\section{Figure Captions:}

Figure 1 Process of creating the spatiotemporal template from a set of recordings for the $\mathrm{N}^{\text {th }}$ pathway. Each column of the matrix corresponds to the recordings from all contacts at a single time step up to the time $T$. The columns are then concatenated to create a single vector corresponding to a spatiotemporal template of the specific pathway being observed.

Figure 2 (a) Cross section of the rat sciatic nerve in the FE model, with different colours representing different types of tissues or materials [20]. (b) Schematic diagram of the matrix nerve cuff, $1 \mathrm{~mm}$ in diameter when rolled. All dimensions are in millimeters [21]

Figure 3 (a) Regions within the fascicles used to model the neural pathways in the $\mathrm{DF}_{\mathrm{a}} \mathrm{DF}_{\mathrm{i}}, \mathrm{DF}_{\mathrm{a}} \mathrm{SF}_{\mathrm{i}}, \mathrm{SF}_{\mathrm{a}} \mathrm{DF}_{\mathrm{i}}$, and $\mathrm{SF}_{\mathrm{a}} \mathrm{SF}_{\mathrm{i}}$ cases. In the $\mathrm{DF}_{\mathrm{a}} \mathrm{DF}_{\mathrm{i}}$ and $\mathrm{DF}_{\mathrm{a}} \mathrm{SF}_{\mathrm{i}}$ cases, 1 of the 3 regions in each fascicle was randomly selected to be used for each fold. In the $\mathrm{SF}_{a} \mathrm{DF}_{\mathrm{i}}$ and $\mathrm{SF}_{\mathrm{a}} \mathrm{SF}_{\mathrm{i}}$ cases, each fascicle was explored and the results averaged over the 3 fascicles for each fold. Spatial variability was introduced by selecting a mesh element within the region for each neural pathway, for each trial. Here the 3 colours - dark blue, red and green - are used to denote the regions for the different neural pathways. For the SFAE case, not illustrated here, fascicles were divided into 2 equal parts (top and bottom).

(b) Shows a simulated neural recording at one of the contacts in the center ring at SNR $=-10 \mathrm{~dB}$. Note: Noise segments have been added to both sides of our simulated recording for illustrative purposes.

Figure 4 Mean classification accuracies for the different test scenarios. All SNRs are in dB. ST - Spatiotemporal templates (our algorithm), BSF - Bayesian Spatial Filters, VSR - Velocity Selective Recording

Figure 5 Mean fraction of spikes missed for the different test scenarios. All SNRs are in dB.

Figure 6 (a) Mean classification accuracy for the SFAE case. (b) Mean fraction of missed spikes for the SFAE case. All SNRs are in $\mathrm{dB}$.

Figure 7 Mean Pearson correlation values for the different test scenarios. All SNRs are in dB. Note: In cases where the Pearson correlation was found to be $\mathrm{NaN}$ (i.e. spikes were all classified to the other classes) the value was set to 0 .

Figure 8 Estimated reconstructed firing rates across various SNRs. (a) $\mathrm{SNR}=\infty \mathrm{dB}$, (b) $\mathrm{SNR}=-10 \mathrm{~dB}$, (c) $\mathrm{SNR}=-$ $15 \mathrm{~dB}$, (d) $\mathrm{SNR}=-25 \mathrm{~dB}$. Instances where the firing rate was above the maximum average rate of $60 \mathrm{~Hz}$ were due to the convolution with the Gaussian kernel when the underlying Poisson process produced closely clustered spikes.

Figure 9 The first plot shows a sub-section of the first fold of the $\mathrm{DF}_{\mathrm{a}} \mathrm{DF}_{\mathrm{i}}$ case with $\mathrm{SNR}=\infty$, the second plot shows the same signal with white Gaussian noise added (SNR -20 dB) and the last 3 plots show the predicted firing patterns of the three pathways based on the application of our algorithm to the noisy data. Note in this figure, one time step is 56 samples apart (each corresponding to one of the 56 channels of the electrode) 
Figure 1

$$
\begin{aligned}
& 1 \text { Tripole Referenced Recordings }
\end{aligned}
$$

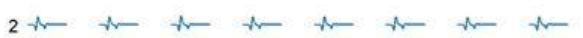

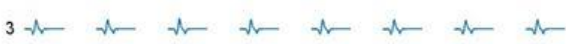

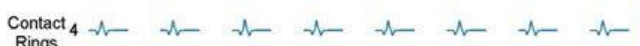

$$
\begin{aligned}
& \text { Rings } 5 \text { th th th th th th th th } \\
& 6 \text { at th th th th th th th }
\end{aligned}
$$

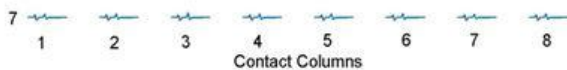

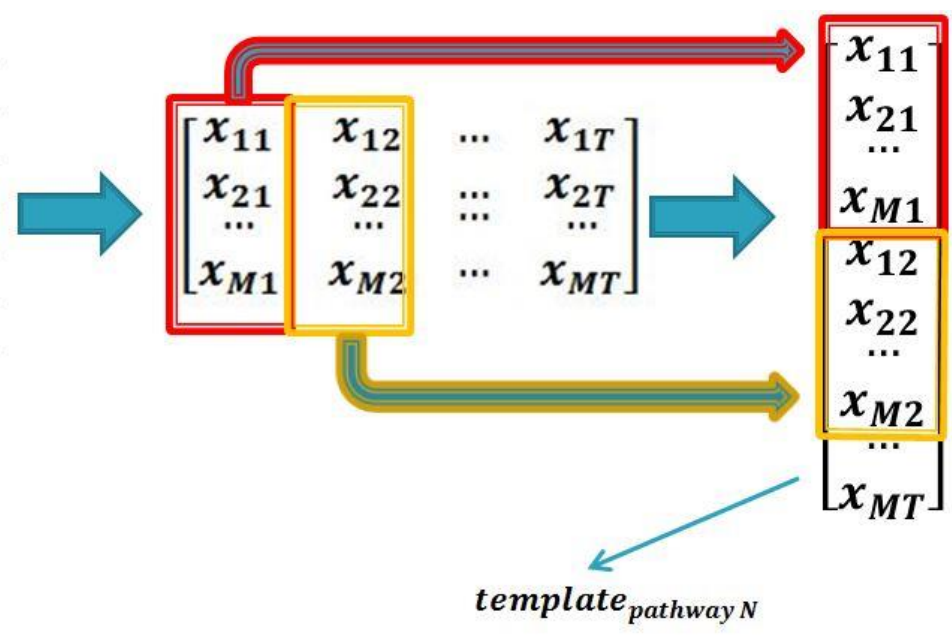

$\mathrm{M} \times \mathrm{T}$ matrix

$M=$ Number of contacts

$\mathrm{T}=$ Number of time steps 
Figure 2

(a)

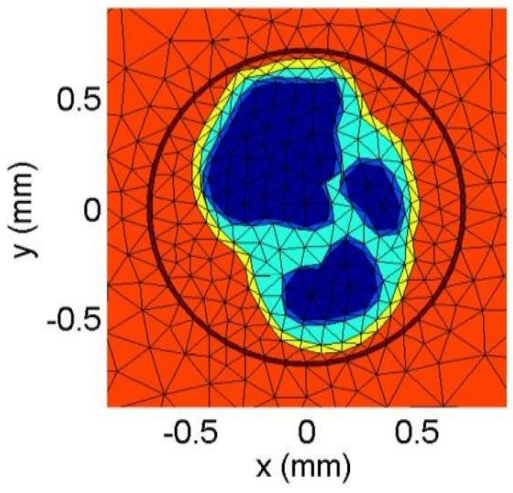

(b)

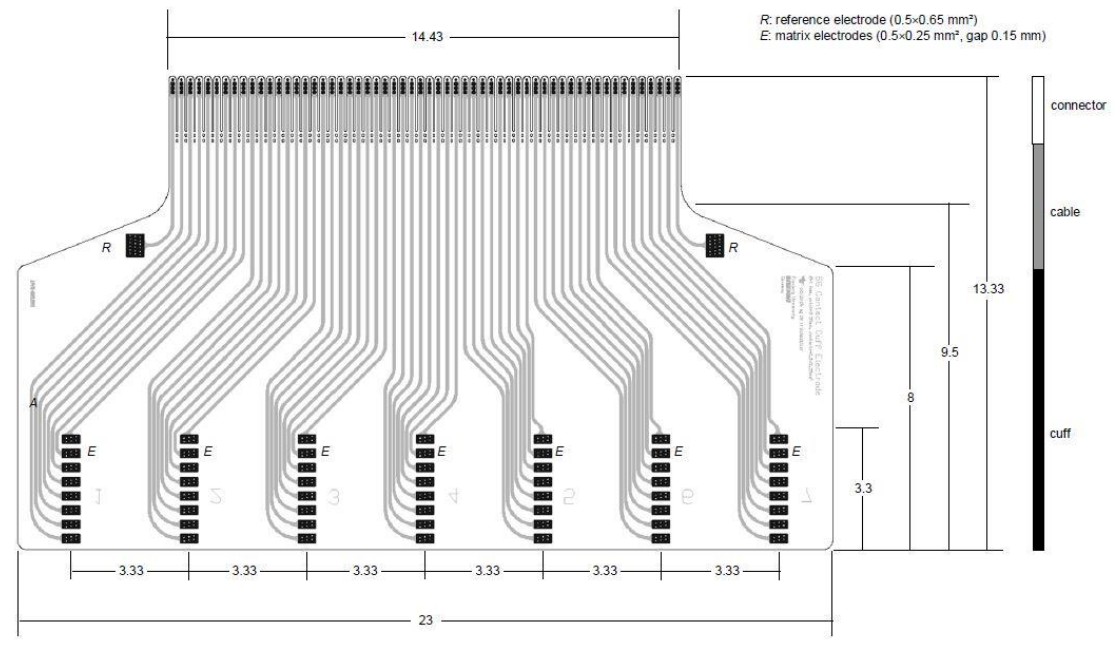


Figure 3

(a)

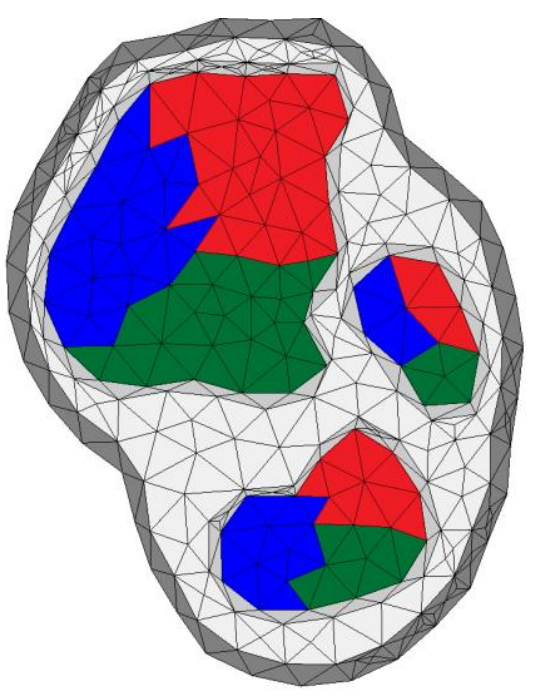

(b)

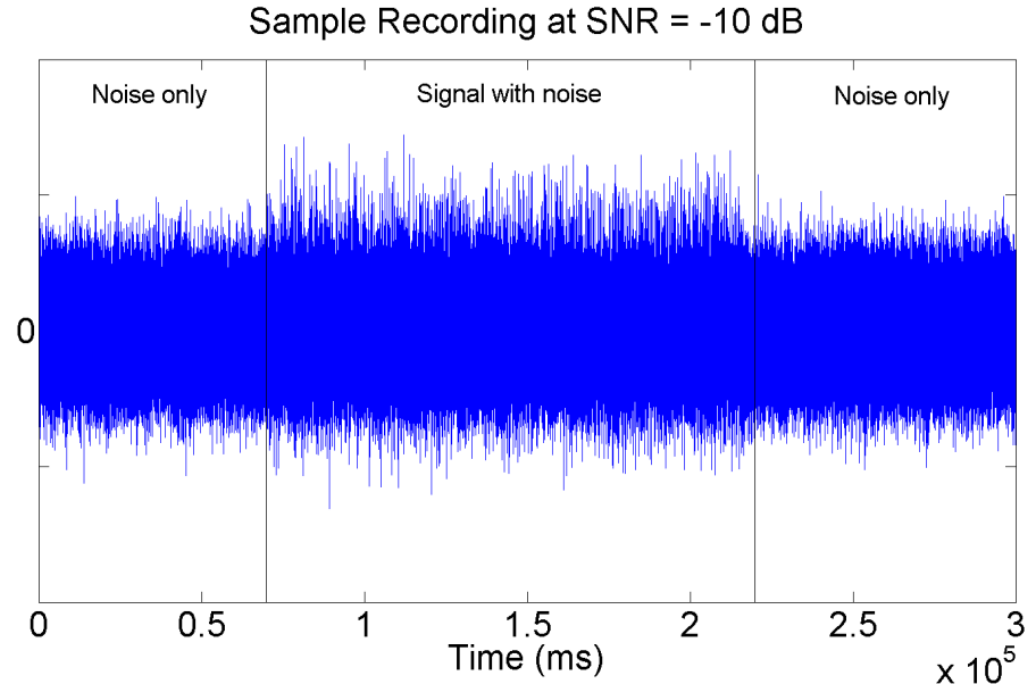


Figure 4

(a)

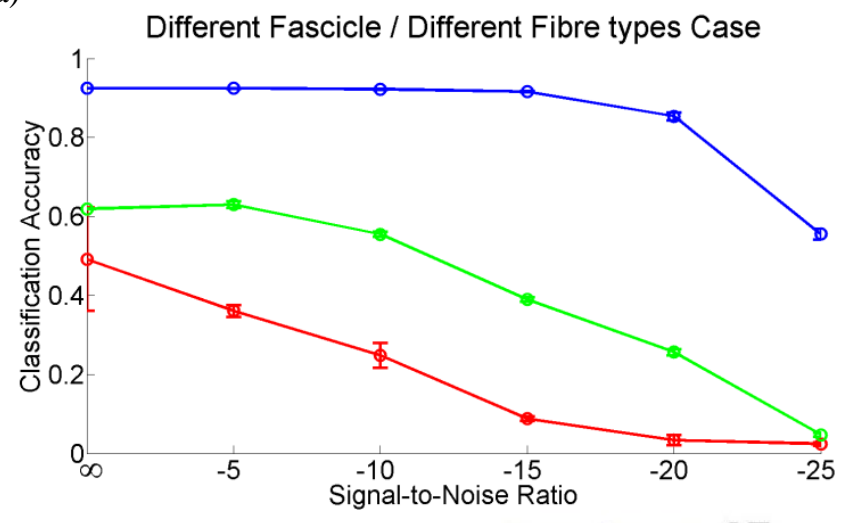

(c)
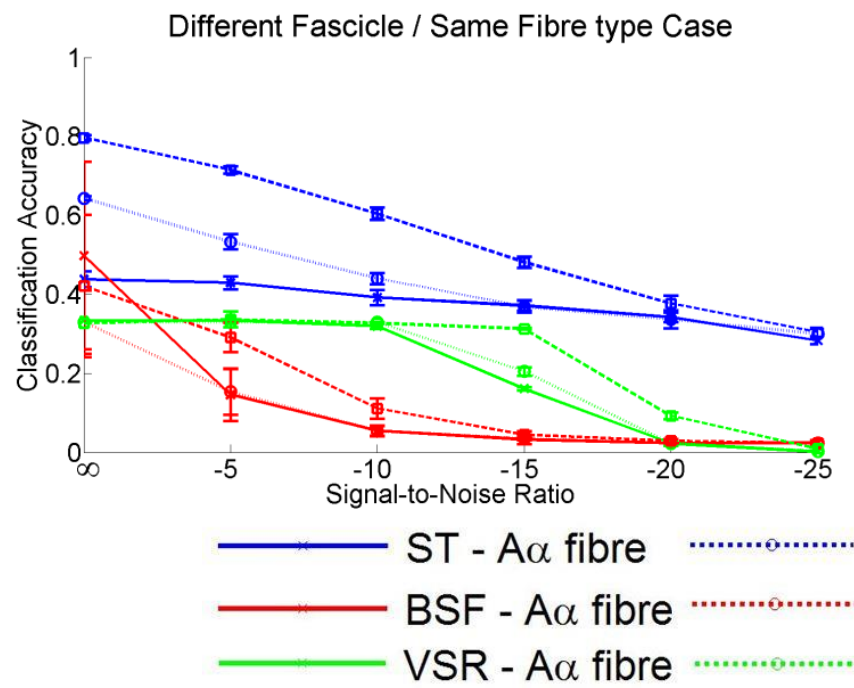

(b)

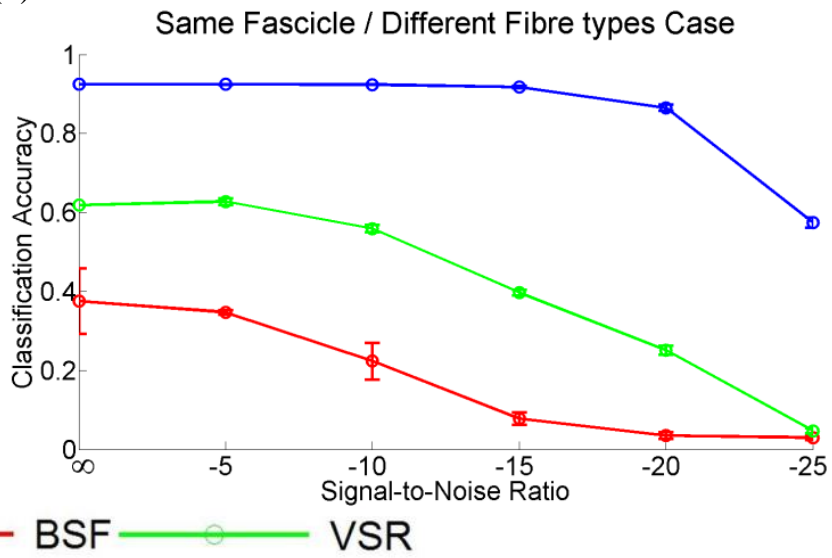

(d)

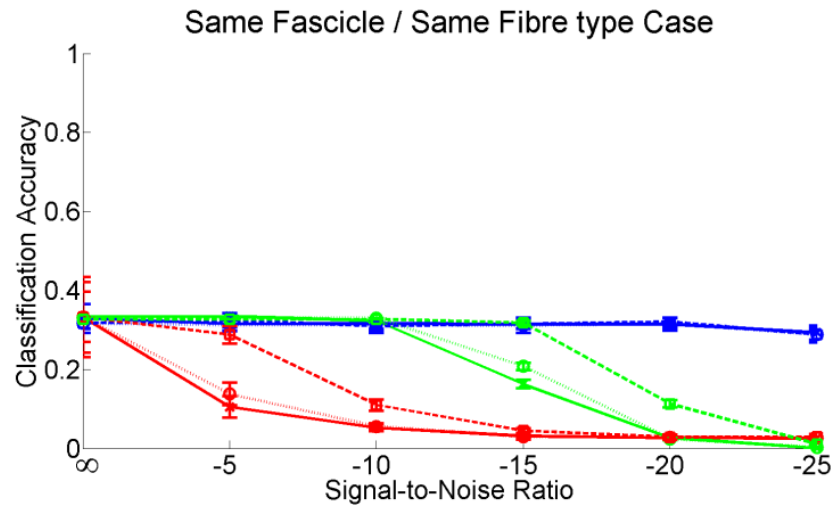

ST - A $\beta$ fibre

ST - A $\delta$ fibre

BSF - A $\beta$ fibre ------- BSF - A $\delta$ fibre

VSR - A $\beta$ fibre 
Figure 5
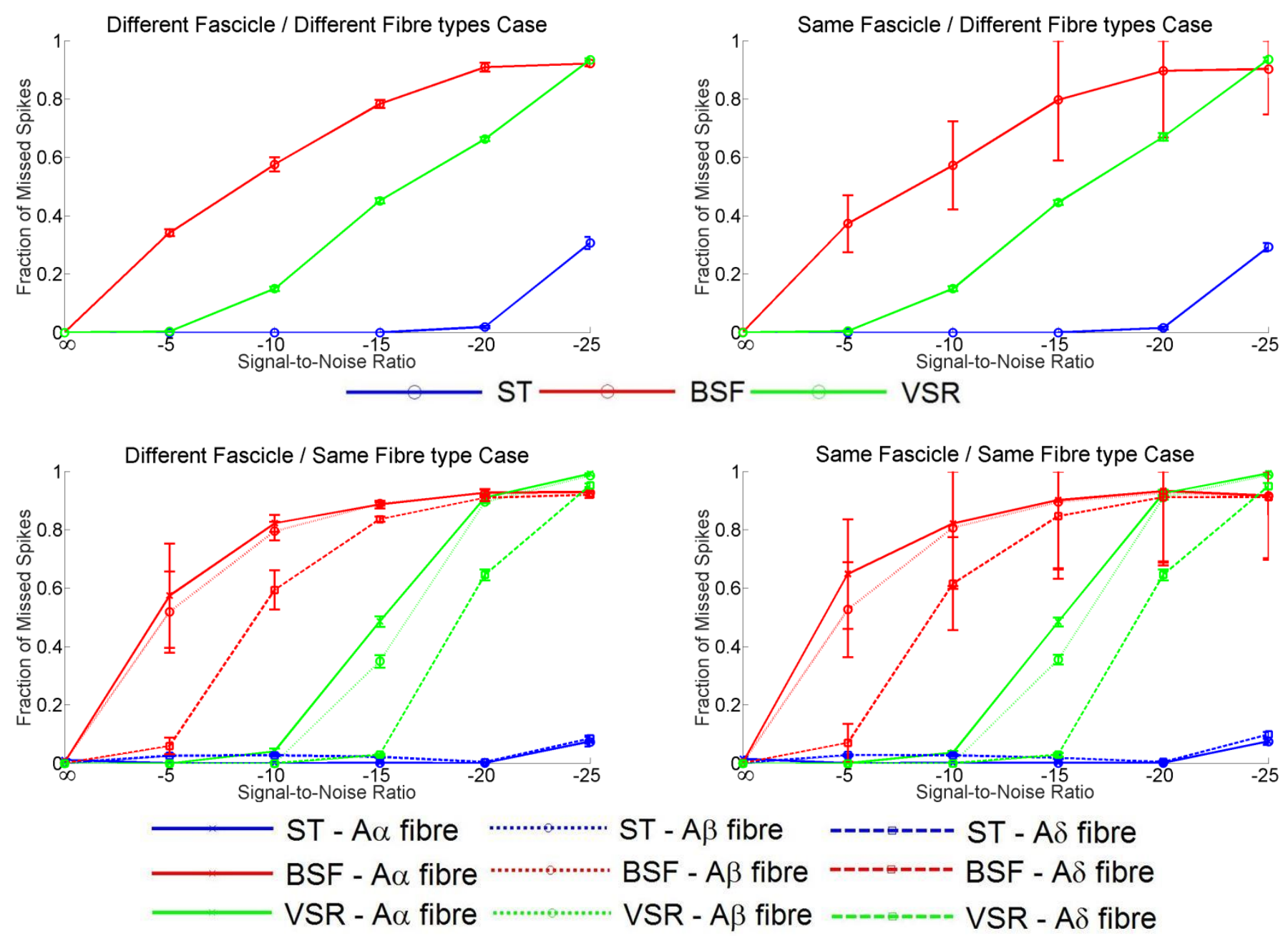

ST - A $\beta$ fibre

ST - A $\delta$ fibre

BSF - A $\beta$ fibre

BSF - A $\delta$ fibre

VSR - A $\beta$ fibre

VSR - A $\delta$ fibre 
Figure 6

(a)
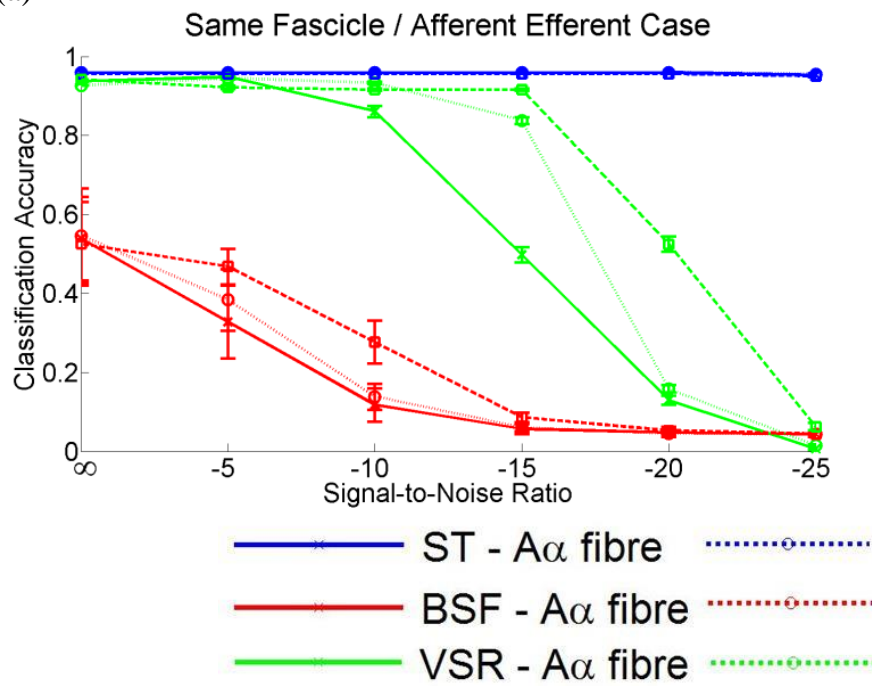

(b)

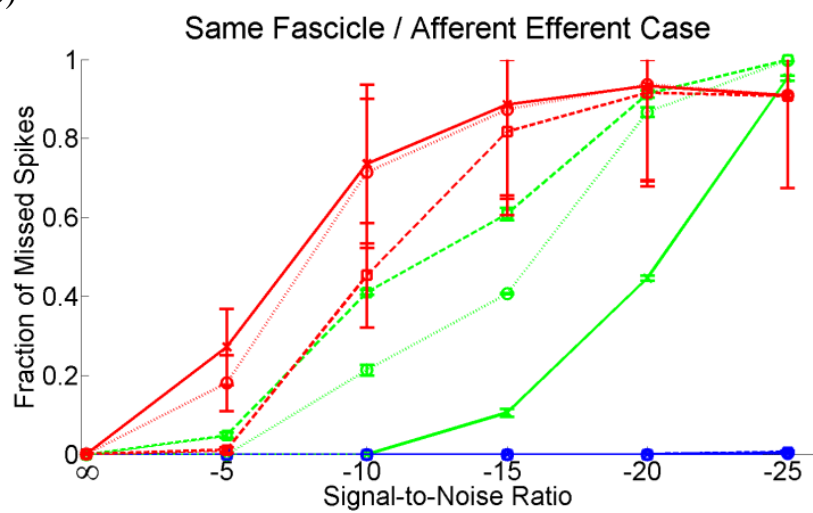

ST - A $\beta$ fibre

TT - A $\delta$ fibre

BSF - A $\beta$ fibre ------- BSF - A $\delta$ fibre

VSR - A $\beta$ fibre -------- VSR - $A \delta$ fibre 
Figure 7
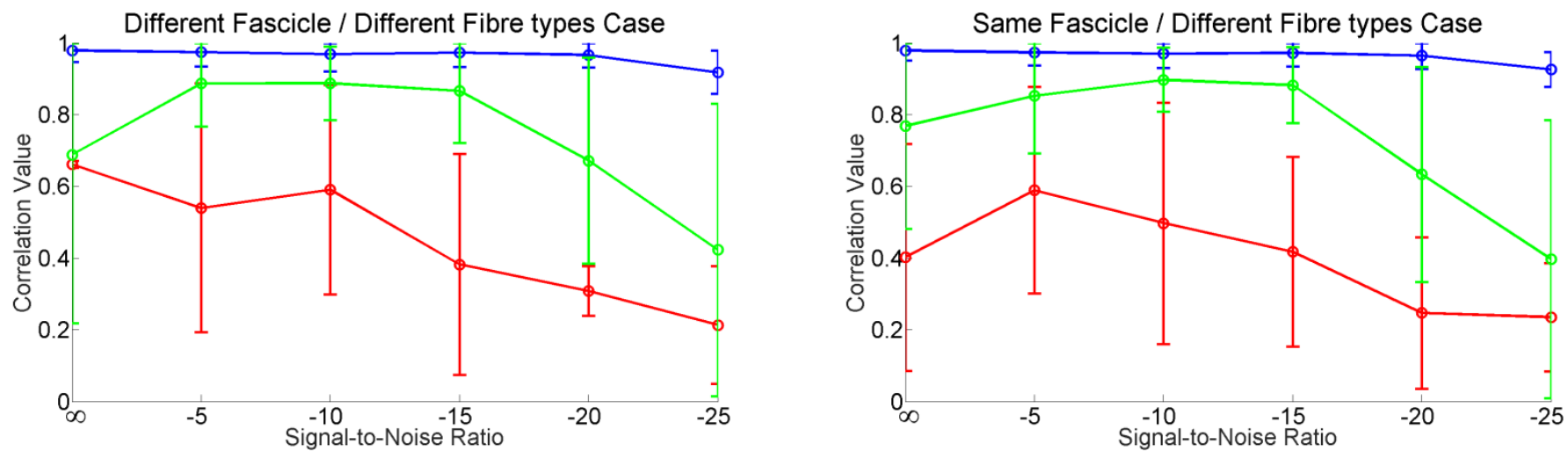

ST

BSF

VSR
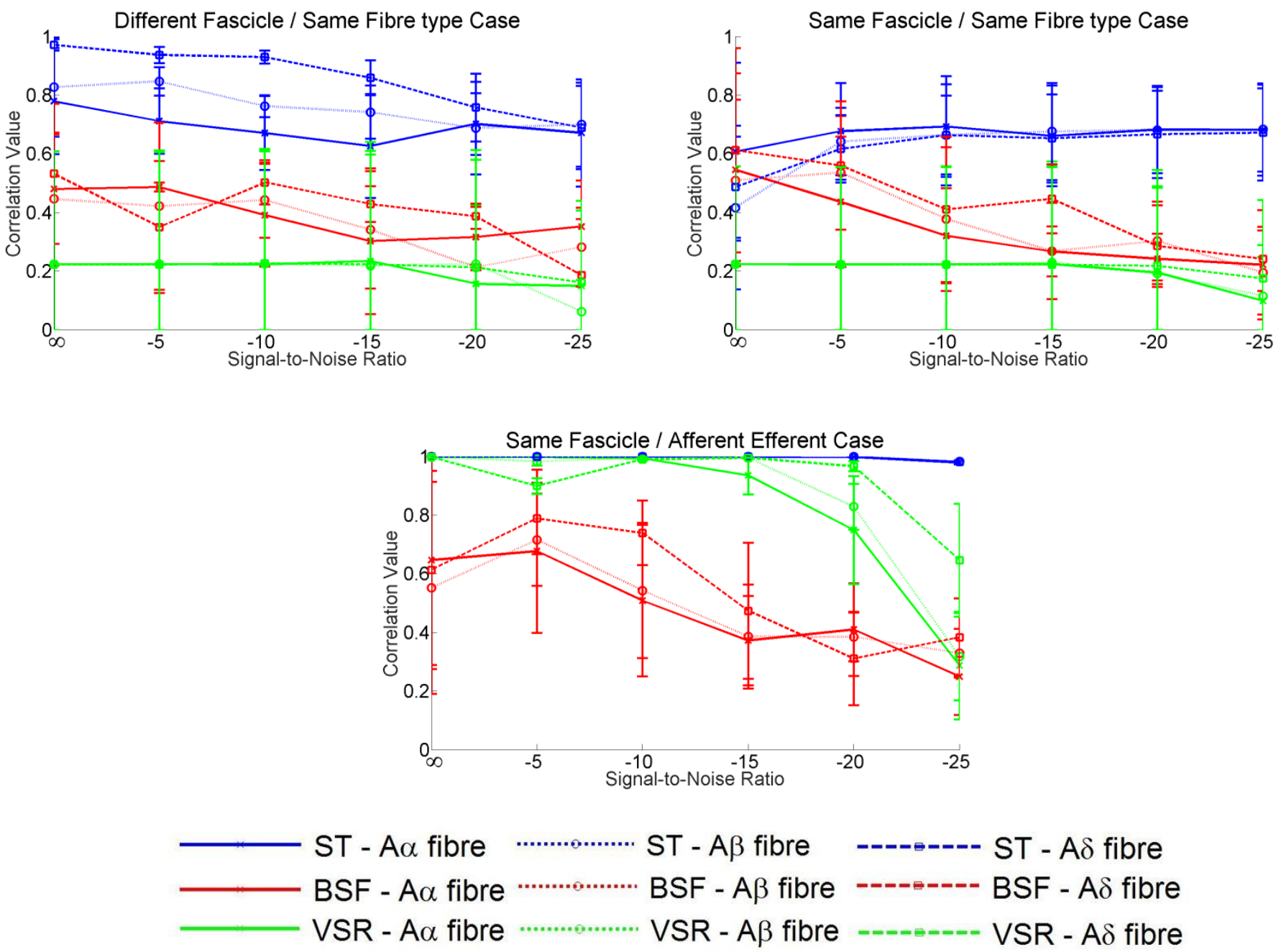
Figure 8

(a)

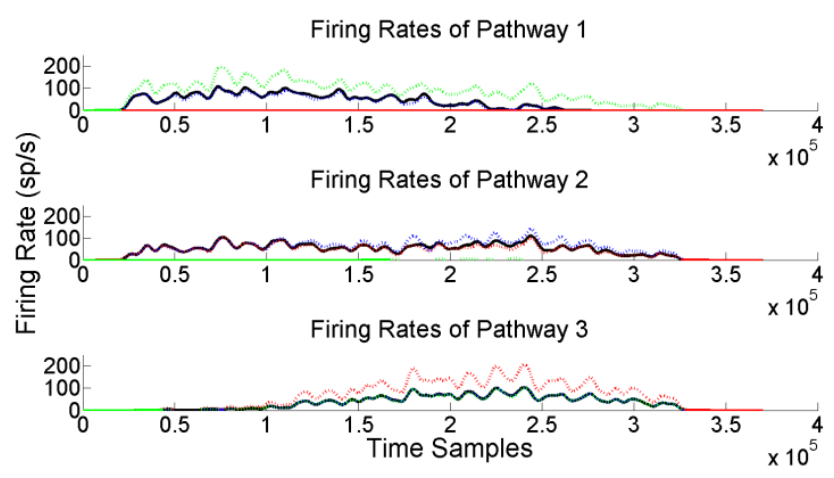

(c)

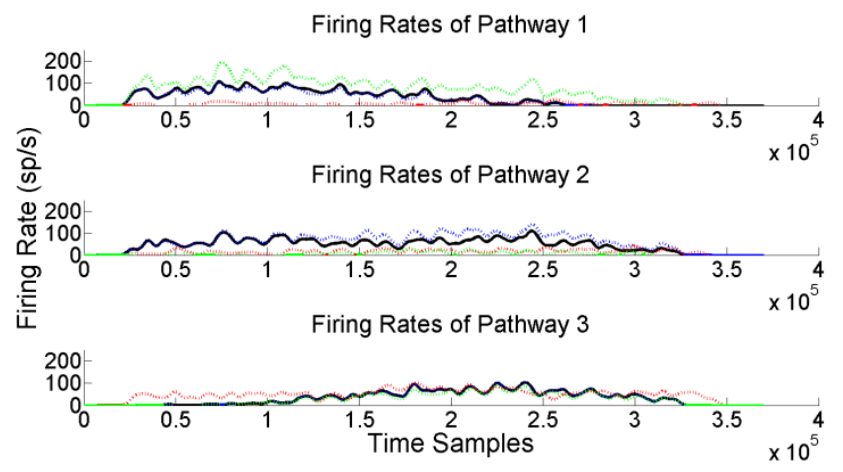

(b)

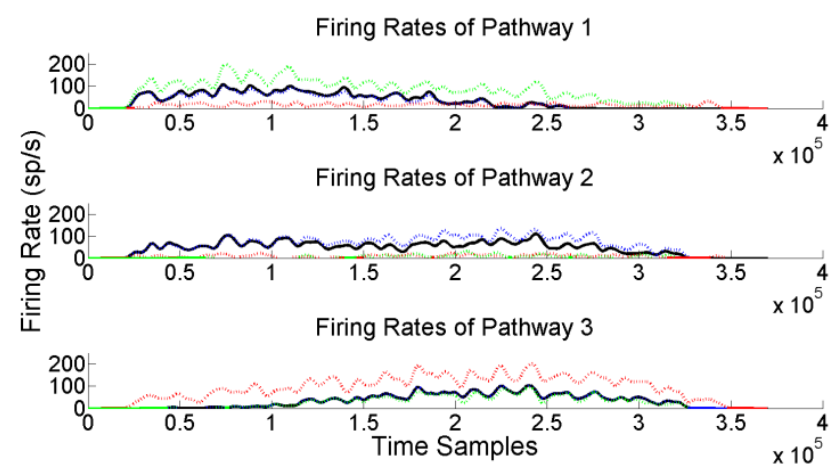

(d)

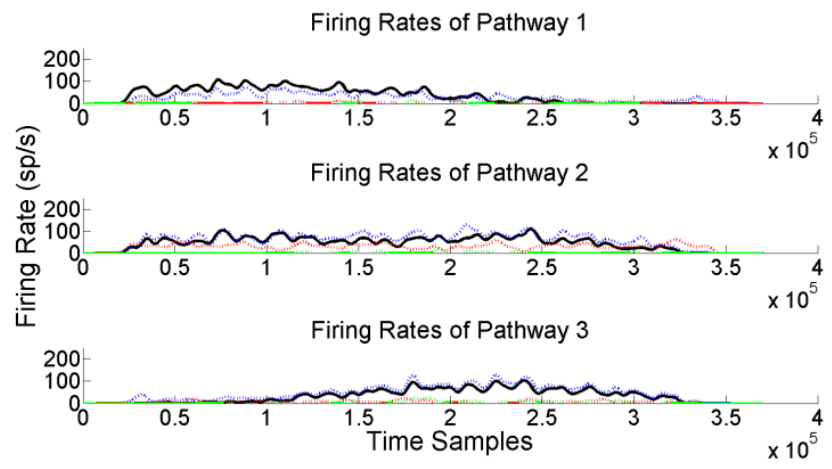


Figure 9

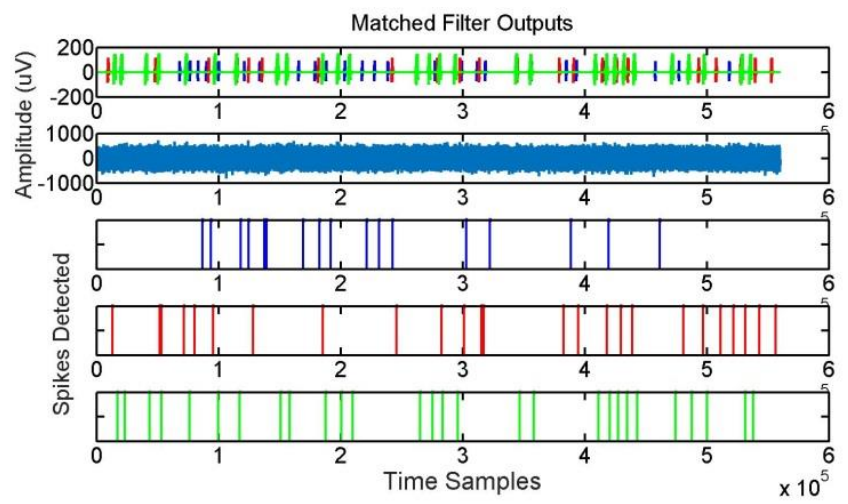

